\section{Apoio social e auto-exame das mamas no Estudo Pró-Saúde}

\author{
Social support and breast self-examination \\ in the Pró-Saúde Study
}

Célia Regina de Andrade 1

Dóra Chor 1

Eduardo Faerstein 2

Rosane Harter Griep 3

Claudia S. Lopes 2

Maria de Jesus Mendes da Fonseca ${ }^{1}$
1 Escola Nacional Saúde Pública, Fundação Oswaldo Cruz, Rio de Janeiro, Brasil.

2 Instituto de Medicina Social, Universidade do Estado do Rio de Janeiro, Rio de Janeiro, Brasil.

3 Escola de Enfermagem Anna Nery, Universidade Federal do Rio de Janeiro, Rio de Janeiro, Brasil.

Correspondência C. R. Andrade Departamento de Epidemiologia e Métodos Quantitativos em Saúde. Escola Nacional Saúde Pública, Fundação Oswaldo Cruz. Rua Leopoldo Bulhõe 1480, Rio de Janeiro, RJ 21041-210, Brasil. celiara@ensp.fiocruz.br

\section{Abstract}

The authors investigated the association between the reported frequency of breast self-examination and five dimensions of social support (material, emotional, affective, information, and positive social interaction) among 2,240 female employees from a university in Rio de Janeiro who were participants in Phase 1 of a cohort study (Pró-Saúde Study). Data were collected through a multidimensional self-administered questionnaire. Among participants, 43\% reported doing breast self-examination "every month", or "almost every month"; $24 \%$ informed they seldom or never practiced self-examination. Participants with the highest scores in the five dimensions of social support reported higher frequency of breast self-examination. In all dimensions, the odds of practicing self-examination were approximately twice as high among women in the upper tertile of social support scores and about 50\% higher among those in the intermediate tertile, as compared to those in the lower tertile of the scores (linear trend test, $p<$ 0.001). The results' consistency suggests that several dimensions of social support can contribute positively to the regular practice of health-related self-care.

Social Support; Breast Self-examination; Women's Health

\section{Introdução}

Estudos epidemiológicos têm evidenciado a importância do ambiente psicossocial na saúde: uma relação inversa entre a intensidade de laços sociais e a mortalidade geral, por exemplo, vem sendo consistentemente observada 1,2,3, e parece ser devida especialmente à redução dos óbitos por doenças cardiovasculares, acidentes e suicídio 4 . Além disso, foram também demonstradas associações inversas entre laços sociais e comportamentos de risco 5 e utilização de serviços de saúde para fins de diagnóstico e tratamento 6 .

Dentre as características dos laços sociais destaca-se, especificamente, o conceito de apoio social, definido por Sherbourne \& Stewart 7 como o grau com que relações interpessoais atendem a determinadas necessidades. Várias hipóteses têm sido propostas em relação aos mecanismos através dos quais o apoio social favoreceria a saúde. Por exemplo, o apoio social poderia exercer efeito tamponador (buffer), moderando os efeitos de eventos estressantes, além de proporcionar ajuda de natureza variada em situações de necessidade 8,9. O apoio social poderia também reforçar a sensação de controle sobre a própria vida, o que teria efeitos positivos diretos e indiretos sobre a saúde 10. Por outro lado, o apoio social percebido tem sido associado ao bem-estar psicológico, a maior grau de satisfação com a vida e auto-estima, e à menor ocorrência de ansiedade 11 . 
Além disso, diversas investigações sugeriram que a disponibilidade de apoio material e afetivo, assim como informação e outras dimensões do apoio social, por exemplo, por parte de familiares e amigos, podem exercer um papel importante no estímulo à adoção e manutenção de hábitos de autocuidado 3,12 .

A relação entre o apoio social e práticas de autocuidado com a saúde feminina tem sido menos estudada 13,14,15. Dentre estas práticas, a realização periódica de auto-exame das mamas era recomendada como estratégia de detecção precoce do câncer de mama por programas governamentais no Brasil até recentemente (Instituto Nacional de Câncer. Câncer: câncer de mama. http://www.inca.org.br/manual/ mama/casos.html, acessado em 25/Mar/2000), inclusive na época em que os dados do presente estudo foram coletados.

As recomendações relativas ao auto-exame das mamas e com o objetivo de screening têm sido progressivamente mais cautelosas 16,17 , sobretudo após a publicação dos resultados de dois ensaios clínicos realizados na Rússia 18 e na China 19 - que não observaram impacto da prática do auto-exame na mortalidade por câncer de mama. O procedimento, entretanto, ainda têm seu lugar no contexto das práticas de autocuidado com a saúde. No Brasil, as recentes Normas e Recomendações do Ministério da Saúde para o Controle do Câncer de Mama 20 (p. 85) recomendam que o SUS "desenvolva ações de educação para o ensinamento da palpação das mamas pela própria mulher como estratégia dos cuidados com o próprio corpo".

Nesta investigação, sugere-se que a prática de auto-exame das mamas, assim recontextualizada, represente um "comportamento marcador" do autocuidado que as mulheres têm com o seu corpo e com a sua saúde. Não foi possível identificar nenhum estudo epidemiológico conduzido em população brasileira sobre as relações entre apoio social e práticas femininas de autocuidado. Consideramos que estudos nesta temática podem auxiliar no entendimento de fatores que influenciam comportamentos positivos em saúde 3 e subsidiar ações que promovam vínculos sociais nos programas de atenção à saúde da mulher 12,21. Com esse propósito, buscou-se identificar a associação entre níveis de apoio social, em cinco diferentes dimensões (material, emocional, afetivo, de informação, e de interação positiva), e a freqüência relatada de auto-exame das mamas.

\section{Metodologia}

\section{Objetivos e população de estudo}

O Estudo Pró-Saúde tem como objetivo geral investigar prospectivamente o papel de determinantes sociais nos padrões de comportamento de saúde e de morbidade física e mental em uma população de funcionários técnicoadministrativos de uma universidade no Rio de Janeiro 22 . As análises aqui apresentadas dizem respeito a 2.240 mulheres $(91 \%$ da população feminina elegível) participantes da primeira fase de coleta de dados (agosto a outubro de 1999) do estudo, e incluem dados colhidos por meio de questionário multidimensional, autopreenchível no ambiente de trabalho. Foram excluídas as funcionárias aposentadas, as cedidas a outras instituições e aquelas licenciadas sem vencimentos. Funcionárias em licença médica foram convidadas a participar do estudo, inclusive com realização de visita domiciliar, se necessário.

\section{Medidas}

\section{- Auto-exame das mamas}

A prática de auto-exame das mamas foi investigada por intermédio da pergunta "Com que freqüência você mesma examina suas mamas (palpação), com o objetivo de descobrir caroços ou qualquer anormalidade?". As categorias de respostas oferecidas foram: "nunca", "raramente", “às vezes”, “quase todo mês" e "todo mês". A freqüência de realização do auto-exame das mamas foi inicialmente analisada com seu agrupamento em três categorias: "todo mês/quase todo mês"; “às vezes" e "raramente/nunca". Em estudo de confiabilidade teste-reteste anterior ao trabalho de campo, estimou-se um coeficiente kappa de 0,82 (IC95\%: 0,74-0,89) para as respostas relacionadas à freqüência do autoexame das mamas, o que é considerado nível quase perfeito 23 .

\section{- Apoio social}

Os processos de decisão e implementação da mensuração do apoio social no Estudo PróSaúde, assim como suas características psicométricas, foram descritos em outras publicações 24,25. Após revisão da literatura, aplicou-se técnica qualitativa de discussão em grupo, na qual utilizamos alguns procedimentos adotados em grupos focais e outros adotados em grupos naturais 26 , para explorar percepções e conceitos de apoio social em nosso meio 24 . 
Tendo em vista as conclusões obtidas com a condução dessas discussões, optou-se por incluir no questionário as questões sobre apoio social utilizadas no Medical Outcomes Study (MOS) 7.

Os itens que compõem esse instrumento, originalmente elaborado em inglês, foram submetidos a um processo de tradução e adaptação para o português e avaliados em cinco etapas de pré-testes e estudo piloto 24 . A confiabilidade teste-reteste da escala variou entre "substancial" (Coeficiente de Correlação Intraclasse-CCIC = 0,75; IC95\%: 0,65-0,83) para a dimensão do apoio de informação e "quase perfeita" (CCIC = 0,89; IC95\%: 0,85-0,93) para a de apoio material, segundo os critérios de Landis \& Koch 23. A consistência interna entre as dimensões, estimada por coeficientes alpha de Cronbach, variou entre 0,75 e 0,91 25 .

Como na versão original, o questionário é composto por 19 itens, abrangendo cinco dimensões funcionais de apoio social 7: material (quatro perguntas - provisão de recursos práticos e ajuda material); afetivo (três perguntas demonstrações físicas de amor e afeto); emocional (quatro perguntas - expressões de afeto positivo, compreensão e sentimentos de confiança); informação (quatro perguntas - disponibilidade de pessoas para a obtenção de conselhos ou orientações) e interação social positi$v a$ (quatro perguntas - disponibilidade de pessoas para divertir-se ou relaxar). Para cada item, o funcionário deveria indicar com que freqüência considerava disponível cada tipo de apoio, em caso de necessidade: nunca, raramente, às vezes, quase sempre e sempre.

Para o cálculo de escores padronizados de cada uma das cinco dimensões de apoio social, foram atribuídos pontos a cada opção de resposta, que variaram entre um (nunca) e cinco (sempre). Os escores foram calculados por meio da soma dos pontos totalizados pelas respostas dadas às perguntas de cada uma das dimensões e divididos pelo número máximo de pontos possível de ser obtido na mesma dimensão. Por exemplo, na dimensão de apoio afetivo, constituída por três perguntas, se uma funcionária respondeu "sempre" em dois itens, e "quase sempre" no terceiro, seu total de pontos foi igual a 14. O resultado dessa adição foi dividido por 15 (número máximo de pontos dessa dimensão) a fim de padronizar os resultados de todas as dimensões, já que estas eram constituídas por diferentes números de perguntas. $\mathrm{O}$ resultado da razão (total de pontos obtidos/ pontuação máxima da dimensão) foi multiplicado por 100. Desse modo, quanto maior o escore, maior o nível de apoio social. As partici- pantes que deixaram de responder a alguma das perguntas foram excluídas do cálculo do escore daquela dimensão; as exclusões variaram entre 0 e $2 \%$, nas cinco dimensões.

\section{Análise dos dados}

Cinco modelos de regressão logística não condicional foram construídos com o objetivo de investigar, em separado, a magnitude da associação entre as diversas dimensões de apoio social e a prática de auto-exame das mamas. Nesses modelos, a variável dependente (prática de auto-exame das mamas) foi dicotomizada ("todo mês/quase todo mês" vs. "às vezes/raramente/nunca") e a exposição foi representada pelos tercis dos escores de cada dimensão de apoio social. Em modelos ajustados foram explorados os possíveis efeitos de confundimento de algumas características; essas variáveis (idade, raça/etnia, escolaridade, religião e local de trabalho) foram selecionadas com base na revisão da literatura, e pelo fato de apresentarem associação $(\mathrm{p}<0,10)$ na análise bivariada, com os escores de apoio social e com a freqüência de auto-exame das mamas em nosso estudo. Além dessas características, explorou-se também, como potencial fator de confusão os escores do General Health Questionnaire (GHQ-12) 27, um instrumento de rastreamento para transtornos mentais comuns amplamente validado e utilizado 28,29 .

Este estudo foi aprovado pelos Comitês de Ética da Universidade e do Hospital Universitário.

\section{Resultados}

A idade das participantes variou entre 22 e 67 anos, com idade média de 41 anos (Tabela 1). Cerca de 50\% das mulheres apresentavam nível universitário; a maioria era casada ou vivia em união (54\%); cerca de $52 \%$ autoclassificaram-se em relação à sua raça/etnia como branca, e $48 \%$ classificaram-se como católicas. Cerca de um terço das participantes relatou renda domiciliar per capita abaixo de quatro salários mínimos da época; na faixa de renda mais alta foram incluídas $25 \%$ do total. Além dessas características, 59\% das mulheres eram funcionárias do hospital universitário.

Do total de respondentes, $44 \%$ informaram realizar o auto-exame das mamas "todo mês" ou “quase todo mês”; 32\%, “às vezes”, e $24 \%$ informaram praticá-lo "raramente" ou "nunca".

A distribuição dos escores das cinco dimensões de apoio social foram semelhantes, com 
Tabela 1

Características sócio-demográficas da população de estudo. Estudo Pró-Saúde, Rio de Janeiro, Brasil, 1999.

\begin{tabular}{|c|c|c|}
\hline & $\mathbf{n}$ & $\%$ \\
\hline \multicolumn{3}{|l|}{ Idade (em anos) } \\
\hline $20-29$ & 214 & 9,6 \\
\hline $30-39$ & 857 & 38,3 \\
\hline $40-49$ & 835 & 37,3 \\
\hline 50 e + & 334 & 14,9 \\
\hline \multicolumn{3}{|l|}{ Escolaridade } \\
\hline Até 1o grau & 444 & 20,2 \\
\hline 2o grau & 742 & 33,7 \\
\hline 3ㅇ grau ou mais & 1.016 & 46,1 \\
\hline \multicolumn{3}{|l|}{ Situação conjugal } \\
\hline Casado/união & 1.158 & 53,8 \\
\hline Separado/divorciado/viúvo & 527 & 24,5 \\
\hline Solteiro & 468 & 21,7 \\
\hline \multicolumn{3}{|l|}{ Raça/etnia } \\
\hline Branca & 1.149 & 51,8 \\
\hline Parda & 606 & 27,3 \\
\hline Preta & 407 & 18,3 \\
\hline Outros (amarela e indígena) & 56 & 2,5 \\
\hline \multicolumn{3}{|l|}{ Religião atual } \\
\hline Católico & 1.057 & 47,7 \\
\hline Evangélico & 338 & 15,2 \\
\hline Espírita & 431 & 19,4 \\
\hline Outras & 195 & 8,8 \\
\hline Não tem religião & 197 & 8,9 \\
\hline \multicolumn{3}{|l|}{$\begin{array}{l}\text { Renda per capita } \\
\text { (em salários mínimos) }\end{array}$} \\
\hline$<4$ & 770 & 36,5 \\
\hline $4-7$ & 816 & 38,6 \\
\hline$\geq 8$ & 526 & 24,9 \\
\hline \multicolumn{3}{|l|}{ Local de trabalho } \\
\hline Campi (fora hospital) & 909 & 41,0 \\
\hline Hospital & 1.307 & 59,0 \\
\hline
\end{tabular}

Nota: as variáveis apresentam totais diferentes de acordo com o número de respondentes.

exceção do apoio afetivo. A média dos escores situou-se em torno de oitenta pontos, e a mediana em torno de 85 pontos. Quanto ao apoio afetivo, a média foi próxima a 86 pontos, e a mediana a 93 pontos.

De modo consistente, foram observadas associações diretas, estatisticamente significativas, entre os tercis de escores das cinco dimensões de apoio social e a prática de auto-exame das mamas, com uma sugestão de gradiente "dose-resposta" linear ( $\mathrm{p}<0,001)$ entre as "exposições” e o desfecho de interesse (Tabela 2). Considerando-se as cinco dimensões, a chance de relatar prática mais freqüente de auto-exame das mamas foi aproximadamente $50 \%$ maior entre mulheres no tercil intermediário, e cerca de duas vezes mais elevada entre aquelas situadas no tercil superior, comparadas às participantes no tercil inferior dos escores de apoio social. Em modelos incluindo as co-variáveis consideradas, as estimativas ajustadas de associação praticamente não diferiram das estimativas não ajustadas, optando-se por apresentar ambas as estimativas.

\section{Discussão}

Entre as mulheres participantes da Fase 1 do Estudo Pró-Saúde, a freqüência de realização do auto-exame das mamas pode ser considerada alta: $43 \%$ das participantes relataram sua prática "todo mês" ou "quase todo mês" - em nível semelhante ao observado entre mulheres em países desenvolvidos, como na Suécia 30 e Estados Unidos 31. Essa semelhança pode ser explicada por duas características que parecem fundamentais para o acesso à informação sobre cuidados com a saúde: alta escolaridade (80\% apresentavam pelo menos o segundo grau completo) e grande parcela constituída por funcionárias da área de saúde $(60 \%$ das mulheres trabalhavam no hospital universitário) 21,32.

Participantes com escores mais altos nas várias dimensões de apoio social relataram, de modo linear e consistente, freqüência mais elevada de auto-exame das mamas. Essa consistência e linearidade dos resultados corroboram, aparentemente pela primeira vez em população brasileira, a hipótese sobre a contribuição positiva do apoio social para a prática regular de autocuidados de saúde, observada em outras populações.

Já foram observadas associações diretas entre o apoio social e a adoção de autocuidados relacionados a comportamentos de saúde, tais como a prática regular de exercícios físicos 33 , o abandono do tabagismo 34 e a dieta saudável 35 .

Mais especificamente em relação às práticas de autocuidado feminino, alguns estudos identificaram o apoio social como preditor importante tanto para a realização periódica do auto-exame das mamas 21,36 - à semelhança de nossa investigação - quanto para a adesão a procedimentos de rastreamento do câncer envolvendo mamografia e exame clínico das mamas 21 . Segundo alguns autores, as mulheres 
Prevalências e razões de chances (RC brutas e ajustadas) da prática de auto-exame das mamas segundo tercis dos escores das dimensões de apoio social. Estudo Pró-Saúde, Rio de Janeiro, Brasil, 1999.

\begin{tabular}{|c|c|c|c|c|c|c|}
\hline \multirow[t]{2}{*}{ Apoio social } & \multicolumn{2}{|c|}{$\begin{array}{c}\text { Pratica auto-exame } \\
\text { das mamas* }\end{array}$} & \multicolumn{2}{|c|}{$\begin{array}{c}\text { Não pratica auto-exame } \\
\text { das mamas }\end{array}$} & \multirow[t]{2}{*}{$\begin{array}{l}\text { RC bruta } \\
\text { (IC95\%) }\end{array}$} & \multirow[t]{2}{*}{$\begin{array}{l}\text { RC ajustada*** } \\
\text { (IC95\%) }\end{array}$} \\
\hline & $n$ & $\%$ & $\mathrm{n}$ & $\%$ & & \\
\hline \multicolumn{7}{|c|}{ Apoio material } \\
\hline $20 a \leq 70$ & 261 & 35,9 & 467 & 64,1 & $1,00-$ & $1,00-$ \\
\hline$\geq 75$ a $\leq 95$ & 370 & 44,3 & 465 & 55,7 & $1,42(1,16-1,75)$ & $1,51(1,22-1,87)$ \\
\hline 100 & 344 & 52,7 & 309 & 47,3 & $1,99(1,60-2,47)$ & $2,08(1,66-2,60)-$ \\
\hline \multicolumn{7}{|l|}{ Apoio afetivo } \\
\hline $20 a \leq 80$ & 270 & 36,6 & 468 & 63,4 & $1,00-$ & $1,00-$ \\
\hline$\geq 87$ a $\leq 93$ & 181 & 40,0 & 271 & 60,0 & $1,16(0,91-1,47)$ & $1,17(0,91-1,50)$ \\
\hline 100 & 524 & 51,1 & 502 & 48,9 & $1,81(1,49-2,20)$ & $1,83(1,49-2,24)$ \\
\hline \multicolumn{7}{|c|}{ Apoio emocional } \\
\hline $20 a \leq 70$ & 255 & 35,0 & 474 & 65,0 & $1,00-$ & $1,00-$ \\
\hline$\geq 75 a \leq 90$ & 314 & 45,2 & 381 & 54,8 & $1,53(1,24-1,90)$ & $1,52(1,21-1,89)$ \\
\hline$\geq 95$ & 406 & 51,3 & 386 & 48,7 & $1,96(1,59-2,40)$ & $1,99(1,60-2,47)$ \\
\hline \multicolumn{7}{|c|}{ Apoio de informação } \\
\hline $20 a \leq 70$ & 256 & 34,8 & 479 & 65,2 & $1,00-$ & $1,00-$ \\
\hline$\geq 75 a \leq 90$ & 309 & 45,4 & 371 & 54,6 & $1,56(1,26-1,93)$ & $1,60(1,28-2,00)$ \\
\hline$\geq 95$ & 410 & 51,2 & 391 & 48,8 & $1,96(1,60-2,41)$ & $1,99(1,60-2,47)$ \\
\hline \multicolumn{7}{|c|}{ Interação positiva } \\
\hline $20 a \leq 70$ & 265 & 35,1 & 491 & 64,9 & $1,00-$ & $1,00-$ \\
\hline$\geq 75 a \leq 90$ & 304 & 45,9 & 359 & 54,1 & $1,57(1,27-1,94)$ & $1,56(1,25-1,95)$ \\
\hline$\geq 95$ & 406 & 50,9 & 391 & 49,1 & $1,92(1,57-2,36)$ & $1,94(1,57-2,40)$ \\
\hline
\end{tabular}

* Pratica auto-exame das mamas: "sempre" ou "quase sempre";

** Não pratica auto-exame das mamas: "nunca", "quase nunca" ou "às vezes";

$\star \star \star$ Ajustada para idade, escolaridade, raça/etnia, religião atual, local de trabalho

e escores de transtorno mental comum (GHQ-12).

são mais influenciadas a adotarem práticas positivas de saúde pela disponibilidade de adequados laços sociais $33,37,38$, fato que pode estar relacionado a diferenças na vulnerabilidade e nos níveis de exposição, determinadas particularmente por diferentes papéis sociais de homens e mulheres na sociedade 38 .

É possível conjecturar um impacto da presença de transtornos mentais e/ou estresse psicológico na associação observada, em nossa população de estudo, entre apoio social e adesão à prática do auto-exame das mamas. Esse impacto teoricamente poderia derivar de relações de confundimento (transtornos mentais ocasionando a diminuição do apoio social percebido) ou por causação comum (transtornos mentais ocasionando ambas as situações: a diminuição do apoio social percebido e da prática de auto-exame das mamas, por exemplo, em razão da diminuição de auto-estima). As rela- ções entre essas condições e características, entretanto, parecem ser bastante complexas, como discutido a seguir.

Por exemplo, embora o apoio social precário seja freqüentemente associado com sintomas depressivos e ansiosos, a direção desta associação nem sempre pode ser demonstrada 3 , apesar de a sugestão de que indivíduos socialmente mais isolados têm um risco aumentado de depressão 39 .

Em outros estudos conduzidos nos Estados Unidos, as evidências sobre essas relações foram pouco consistentes. Kash et al. 40 observaram, entre mulheres com história familiar de câncer de mama, que aquelas que relataram maiores barreiras para a realização de screening e com menos apoio social apresentavam níveis significativamente mais elevados de estresse psicológico, e que a presença de ansiedade relacionava-se diretamente com baixa 
adesão ao auto-exame das mamas. Por outro lado, em estudo conduzido entre mulheres negras, Lyons et al. 41 não encontraram associação entre depressão e freqüência do auto-exame das mamas. Por sua vez, Lerman et al. 42 , em estudo entre mulheres jovens com risco elevado de câncer de mama, relataram que o estresse psicológico apresentou-se significativamente associado tanto com um excesso quanto com um déficit na freqüência de auto-exame das mamas. Em nossa população de estudo, a inclusão nos modelos multivariados da presença de transtorno mental comum (medida por intermédio dos escores do GHQ-12) não modificou as estimativas de associação entre apoio social e a prática de auto-exame das mamas.

Em nosso estudo, avaliações conduzidas a respeito da qualidade do processo de mensuração com o questionário utilizado sugeriram alta qualidade dos dados colhidos 22,24 , minimizando a possibilidade de que as associações encontradas sejam atribuídas a erros de mensuração. Especificamente, altos níveis de confiabilidade foram observados em relação às informações sobre os níveis de apoio social e sobre a prática de auto-exame das mamas 25,43 ,

\section{Resumo}

Os autores investigaram a associação entre a freqüência relatada do auto-exame das mamas e cinco dimensões de apoio social (material, emocional, afetivo, de informação e interação social positiva) entre 2.240 funcionárias de uma universidade no Rio de Janeiro, participantes da Fase 1 de um estudo de coorte (Estudo Pró-Saúde). A coleta de informações foi feita por intermédio de um questionário autopreenchível. A realização do auto-exame das mamas "todo mês" ou "quase todo mês" foi relatada por 43\% das funcionárias; $24 \%$ informaram praticá-lo "raramente" ou "nunca". As participantes com escores mais altos nas cinco dimensões de apoio social relataram freqüência mais elevada de auto-exame das mamas. Em todas as dimensões, a chance de praticar o auto-exame foi aproximadamente duas vezes maior entre aquelas situadas no tercil superior, e cerca de 50\% mais elevada entre aquelas no tercil intermediário, quando comparadas às participantes situadas no tercil inferior dos escores de apoio social (teste para tendência linear: $p<$ 0,001 ). A consistência dos resultados sugere que diversas dimensões de apoio social podem contribuir positivamente para a prática regular de autocuidados de saúde.

Apoio Social; Auto-exame de Mama; Saúde da Mulher variando de substancial a quase perfeita, segundo os critérios de Landis \& Koch ${ }^{23}$. Além disso, avaliações de validade de constructo da escala de apoio social evidenciaram que o referido instrumento, traduzido e adaptado para o português, manteve propriedades psicométricas adequadas quando utilizado na população do Estudo Pró-Saúde 44.

As análises aqui apresentadas são baseadas em informações colhidas transversalmente (na Fase 1 do estudo) sobre o apoio social percebido e a prática usual de auto-exame das mamas. Neste caso, contudo, a causalidade reversa, isto é, a influência da prática da auto-exame nos níveis de apoio social, possível em estudos transversais, careceria de significado teórico ou prático.

Nossos resultados sugerem a importância do apoio social no estímulo a práticas de autocuidado de saúde da mulher. Tendo em vista a natureza longitudinal do Estudo Pró-Saúde e as associações observadas nesta investigação, análises futuras poderão contribuir para uma maior compreensão sobre a associação do apoio social com essas práticas e em outros comportamentos e desfechos relacionados à saúde.

\section{Colaboradores}

C. R. Andrade contribuiu na coleta de dados do Estudo Pró-Saúde, participou da construção do indicador de apoio social, análise, revisão bibliográfica e discussão dos resultados. D. Chor participou do planejamento e implementação do Estudo Pró-Saúde, revisão bibliográfica, construção do indicador de apoio social, análise e discussão dos resultados. E. Faerstein participou do planejamento e implementação do Estudo Pró-Saúde, revisão bibliográfica, análise e discussão dos resultados. R. H. Griep participou da revisão bibliográfica, construção do indicador de apoio social, análise e discussão dos resultados. C. S. Lopes participou do planejamento e implementação do Estudo Pró-Saúde e discussão dos resultados. M. J. M. Fonseca participou da análise de dados e discussão dos resultados. Todos os autores participaram da elaboração do artigo final. 


\section{Referências}

1. Penninx WB, van Tilburg T, Kriegsman DM, Deeg DJ, Boeke AJ, van Eijk JT. Effects of social support and personal coping resources on mortality in older age: The Longitudinal Aging Study, Amsterdam. Am J Epidemiol 1997; 146:510-9.

2. Dalgard OS, Haheim LL. Psychosocial risk factors and mortality: a prospective study with special focus on social support, social participation and locus of control in Norway. J Epidemiol Community Health 1998; 52:476-81.

3. Berkman LF, Glass T. Social integration, social networks, social support and health. In: Berkman LF, Kawachi I, editors. Social epidemiology. New York: Oxford University Press; 2000. p. 137-73.

4. Kawachi I, Colditz G, Ascherio A, Rimm EB, Giovannucci $\mathrm{E}$, Stampfer MJ, et al. A prospective study of social networks in relation to total mortality and cardiovascular disease in men in the USA. J Epidemiol Community Health 1996; 50:245-51.

5. Dressler WW, Balieiro MC, Santos JE. The cultural construcion of social support in Brazil: associations with health outcomes. Cult Med Psychiatry 1997; 21:303-35.

6. Kouzis AC, Eaton WW. Absence of social networks, social support and health services utilization. Psychosom Med 1998; 28:1301-10.

7. Sherbourne CD, Stewart AL. The MOS social support survey. Soc Sci Med 1991; 36:705-14.

8. Cobb S. Social support as a moderator of life stress. Psychosom Med 1976; 38:300-14.

9. Cohen S, Wills TA. Stress, social support and the buffering hypothesis. Psychol Bull 1985; 98:310-57.

10. Rodin J. Aging and health: effects of the sense of control. Science 1986; 233:1271-6.

11. Broadhead WE, Gehlbach SH, DeGruy FV, Kaplan BH. The DUKE-UNC Functional Social Support Questionnaire: measurement of social support in family medicine patients. Med Care 1988; 26:709-21.

12. Taylor SE, Repettu RL, Seeman T. Health psychology: what is an unhealthy environment and how does it get under the skin? Annu Rev Psychol 1997; 48:411-47.

13. Umberson D. Gender, marital status and the social control of health behavior. Soc Sci Med 1992; 34:907-17.

14. Kang SH, Bloom JR. Social support and cancer screening among older black Americans. J Natl Cancer Inst 1993; 85:737-42.

15. Suarez L, Ramirez AG, Villareal R, Marti J, McAlister A, Talavera GA, et al. Social networks and cancer screening in four U.S. Hispanic groups. Am J Prev Med 2000; 19:47-52.

16. U.S. Preventive Services Task Force. Guide to clinical preventive services. 2nd Ed. Washington DC: U.S. Department of Health and Human Services; 1996.

17. Nekhlyudov L, Fletcher SW. Is it time to stop teaching breast self-examination? CMAJ 2001; 164:1851-2.

18. Semiglazov VF, Moiseenko VM, Manikhas AG, Protsenko AS, Kharikova, RS, Popova RT, et al. Interim results of a prospective randomizaded study of self-examination for early detection of breast cancer, Russia, St. Petersburg. Vopr Onkol 1999; 45:265-71.
19. Thomas DB, Gao DL, Ray RM, Wang WW, Allison CJ, Chen FL, et al. Randomized trial of breast selfexamination in Shanghai: final results. J Natl Cancer Inst 2002; 94:1445-57.

20. Ministério da Saúde. Normas e recomendações do Ministério da Saúde para o controle do câncer de mama - documento de consenso. Rev Bras Cancerol 2004; 50:77-90.

21. Katapodi MC, Facione NC, Miaskowski C, Dodd MJ, Waters C. The influence of social support on breast cancer screening in a multicultural community sample. Oncol Nurs Forum 2002; 29:845-52.

22. Faerstein E, Lopes CS, Valente K, Plá MAS, Ferreira MB. Pré-teste de um questionário multidimensional autopreenchível: a experiência do Estudo Pró-Saúde. Physis (Rio J) 1999; 9:117-30.

23. Landis JR, Koch GG. The measurement of observer agreement for categorical data. Biometrics 1977; 33:159-74.

24. Chor D, Griep RH, Lopes CS, Faerstein E. Medidas de rede e apoio social, o Estudo Pró-Saúde: pré-testes e estudo piloto. Cad Saúde Pública 2001; 17:887-96.

25. Griep RH, Chor D, Faerstein E, Lopes CS. Apoio social: confiabilidade teste-reteste de escala no Estudo Pró-Saúde. Cad Saúde Pública 2003; 19: 625-34.

26. Coreil J. Group interview methods in community health research. Med Anthropol 1995; 16:193-210.

27. Goldeberg DP, Blackwell B. Psychiatric illness by questionnaire. London: Oxford University Press; 1970.

28. Mari JJ, Williams P. A comparison of the validity of two psychiatric screening questionnaires (GHQ12 and SRQ-20) in Brazil, using Relative Operating Characteristic (ROC) analysis. Psychol Med $1985 ; 15: 651-9$.

29. Stanfeld AS, Marmot MG. Social class and minor psychiatric morbidity. A validated screening survey using the General Health Questionnaire in British civil servants. Psychol Med 1992; 22:73949.

30. Persson K, Svensson PG, Ek AC. Breast self-examination: an analysis of self-reported practice. J Adv Nurs 1997; 25:886-92.

31. Gray ME. Factors related to practice of breast selfexamination in rural women. Cancer Nurs 1990; 13:100-7.

32. Mickey RM, Durski J, Worden JK, Danigelis NL. Breast cancer screening and associated factors for low-income African-American women. Prev Med 1995; 24:467-76

33. Treiber FA, Baranowski T, Braden DS, Strong WB, Levy M, Knox W. Social support for exercise: relationship to physical activity in young adults. Prev Med 1991; 20:737-50.

34. Hanson B, Isacsson S, Janzon L, Lindell S. Social support and quitting smoking for good: is there an association? Results from the population study "Men born in 1914", Malino, Sweden. Addict Behav 1990; 15:221-33.

35. Kelsey K, Earp JAL, Kirkley BG. Is social support beneficial for dietary change? A review of the literature. Fam Community Health 1997; 20:70-82. 
36. Wagle A, Komorita NI, Lu Z-Y. Social Support and breast self-examination. Cancer Nurs 1997; 20:42-8.

37. Kandrack MA, Grant KR, Segall A. Gender differences in health related behaviour: some unanswered questions. Soc Sci Med 1991; 32:579-90.

38. Molinari C, Ahern M, Hendryx M. The relationship of community quality to the health of women and men. Soc Sci Med 1998; 47:1113-20.

39. Murphy E. Social origins of depression in old age. Br J Psychiatry 1982; 141:135-42.

40. Kash KM, Holland JC, Halper MS, Miller DG. Psychological distress and surveillance behaviors of women with a family history of breast cancer. J Natl Cancer Inst 1992; 84:24-30.

41. Lyons E, Zhu K, Hunter S, Bernard L, Payne-Wilks $\mathrm{K}$, Roland C, et al. Depression-related variables and breast self-examination in single older africanamerican women. Ann Epidemiol 2000; 10:463.
42. Lerman C, Kash K, Stefanek M. Younger women at increased risk for breast cancer: perceived risk, psychological well-being, and surveillance behavior. J Natl Cancer Inst Monogr 1994; 16:171-6.

43. Andrade CR. Associação entre apoio social e freqüência relatada de auto-exame das mamas no Estudo Pró-Saúde [Dissertação de Mestrado]. Rio de Janeiro: Escola Nacional de Saúde Pública, Fundação Oswaldo Cruz; 2001.

44. Griep RH. Confiabilidade e validade de instrumentos de medida de rede social e apoio social utilizados no Estudo Pró-Saúde [Tese de Doutorado]. Rio de Janeiro: Escola Nacional de Saúde Pública, Fundação Oswaldo Cruz; 2003.

Recebido em 29/Out/2003

Versão final reapresentada em 31/Ago/2004

Aprovado em 03/Set/2004 